\title{
A FORMAÇÃO DOS TRABALHADORES TÉCNICOS DE SAÚDE COMO OBJETO DE ESTUDO NOS PROGRAMAS DE PÓS-GRADUAÇÃO STRICTU SENSU, NO BRASIL (2002-2016)
}

\author{
Thais Paulo Costa ${ }^{1}$ e Lenina Lopes Soares Silva ${ }^{2}$ \\ ${ }^{1}$ tpaulotc@gmail.com, ${ }^{2}$ leninasilva@hotmail.com \\ Instituto Federal de Educação, Ciência e Tecnologia do Rio Grande do Norte
}

Artigo submetido em outubro/2016 e aceito em dezembro/2016

DOI: $10.15628 /$ rbept.2016.5311

\section{RESUMO}

A história da Educação Profissional em Saúde, no Brasil, tem como grande marco a década de 1980, inserida nas lutas sociais com a finalidade de conquistar um maior reconhecimento para os trabalhadores de nível técnico, considerando que estes atuavam nos serviços de saúde com precária qualificação e sem reconhecimento profissional. A criação das Escolas Técnicas de Saúde pode ser considerada como uma das principais conquistas, destacando-se o seu papel social e político. Entretanto, as formações desses trabalhadores ainda se encontram em um cenário associado à forte flexibilização e precarização das relações de trabalho que tendem a afetar as formações da subjetividade e o reconhecimento social desses profissionais. Este artigo apresenta como objetivo fazer um levantamento da produção do conhecimento sobre a Formação Técnica em Saúde nos Programas de Pós-Graduação Strictu Sensu, no Brasil, no período de 2002 a 2016, tendo como objetivo específico apresentar os procedimentos metodológicos adotados pelos autores nessas produções. A pesquisa foi desenvolvida no mês de agosto de 2016 no repositório do Portal da Coordenação e Aperfeiçoamento de Pessoal de Nível Superior (CAPES). Os resultados apresentaram nove dissertações de mestrado: três de caráter exploratório, quatro estudos de caso, uma pesquisa documental, uma pesquisa avaliativa e uma tese que utilizou a análise documental como procedimento metodológico.

PALAVRAS-CHAVE: Escola Técnica do SUS, Formação Técnica em Saúde, Produção do Conhecimento.

\section{THE FORMATION OF TECHNICAL WORKERS HEALTH AS AN OBJECT OF STUDY IN GRADUATE PROGRAMS STRICTU SENSU, IN BRAZIL (2007-2015)}

\begin{abstract}
The history of Professional Education in Health in Brazil has the milestone the 1980s, set in the social struggles in order to gain greater recognition to the technical level of workers, whereas those worked in health services in poor qualification and no professional recognition. The creation of the Health Technical Schools can be considered as one of the major achievements, especially its social and political role. However, the training of these workers still are in a scenario associated with strong flexibility and precariousness of labor relations that tend to affect the formation of subjectivity and social recognition of these professionals. This article has as objective to survey the production of knowledge on Technical Education in Health in the Graduate Programs
\end{abstract}

Strictu Sensu in Brazil from 2002 to 2016, with the specific objective to present the methodological procedures adopted by the authors in these productions. The research was conducted in August 2016 in the Portal repository of Coordination of Higher Education Personnel (CAPES). The results showed nine dissertations: Three exploratory, four case studies, desk research, an evaluation research and a thesis that used document analysis as a methodological procedure.

KEYWORDS: School SUS Technical, Technical Training in Health, Knowledge Production 


\section{INTRODUÇÃO}

No Brasil, a história da Educação Profissional em Saúde tem como grande marco os anos de 1980, período caracterizado por lutas sociais para maior reconhecimento dos trabalhadores de nível técnico, considerando que esses atuavam nos serviços de saúde com precária qualificação e sem reconhecimento profissional. Essa assertiva é corroborada por Pronko (2011).

As reinvindicações por espaço e formação para essa categoria profissional que se insere na grande classe que vive do trabalho desencadeou conquistas que contribuíram para a formação e reconhecimento dos trabalhadores em seu espaço de trabalho. Iniciativas como o Programa de Preparação Estratégica de Pessoal em Saúde (PREPS), ocorrido no final da década de 1970, foram fundamentais para a criação do Projeto de Formação em Larga Escala de Pessoal de Nível Médio, conhecido como Projeto Larga Escala. Este contribuiu para o processo de ensino e de aprendizagem nos serviços do setor de saúde, ou à educação profissional em saúde. Isso porque a formação nesse projeto considera o ambiente de trabalho como objeto e espaço/campo de estudo e lócus no qual o trabalhador em saúde poderia intervir diretamente por meio de uma formação qualificada em suas ações laborais.

Questiona-se sobre quais procedimentos metodológicos estão sendo utilizados para se compreender a formação técnica em saúde, principalmente naquelas pesquisas que tem como campo empírico as Escolas Técnicas de Saúde? Reconhece-se que essas Escolas foram constituídas com o objetivo de reconhecer o trabalho e garantir a formação dos trabalhadores técnicos em saúde e, nesse sentido, se constituem como um rico campo de produção do conhecimento. Campo que pode ser complementado quando se discute os procedimentos metodológicos utilizados para a discussão dos resultados de estudos que tem a formação dos trabalhadores técnicos em saúde como objetivo.

Frente a esse contexto, este artigo tem como principal objetivo fazer um levantamento da produção de conhecimento acerca da Formação Técnica em Saúde, produzida nos Programas de Pós-Graduação Strictu Sensu no Brasil, no período de 2002 a 2016, e como objetivo específico: caracterizar os procedimentos metodológicos adotados nessas produções para compreender a temática construída em torno dessa formação, visualizando aqueles estudos que têm como campo empírico as Escolas Técnicas de Saúde.

Tal pesquisa busca contribuir com o mapeamento e fortalecimento da produção do conhecimento acerca da educação profissional em saúde em suas especificidades e singularidades por se constituir dentro das Escolas Técnicas de Saúde, notadamente, como uma formação técnica em serviço.

\section{REVISÃO BIBLIOGRÁFICA}

O Projeto Larga Escala contribuiu para a formação dos trabalhadores da saúde envolvidos na construção de um sistema de saúde de caráter universal e integral, tendo como intuito capacitar o pessoal de nível médio em "larga escala" para atender às necessidades do aumento da demanda por atendimento no setor saúde no Brasil.

Em 1986, reconhecendo as ações desenvolvidas pelo Projeto de Formação em Larga Escala, o plenário da Conferência Nacional de Recursos Humanos para a Saúde aprovou diversas propostas, 
dentre elas, a criação de escolas técnicas públicas de caráter multiprofissional e específica para o setor de saúde. Este Projeto, segundo Castro (2008, p.158),

Nasceu da necessidade de equacionar dois graves problemas com os quais se defrontavam os serviços de saúde e o sistema educacional: a inade quabilidade deste último, no sentido de integrar teoria e prática; e a incapacidade do primeiro, em promover programas de formação de pessoal, que fossem além das usuais propostas de treinamento, fragmentadas e pontuais.

Entretanto, o contexto geral no qual as Escolas Técnicas de Saúde foram criadas tencionou ainda mais o desafio de garantir uma formação emancipatória aos trabalhadores envolvidos nos serviços públicos de saúde oferecidos à população brasileira. Na década de 1990, período marcado pelo neoliberalismo no Brasil, são desenvolvidas novas formas de contratação, que associadas à forte flexibilização e precarização das relações de trabalho, tendiam a afetar a formação da subjetividade e do reconhecimento social desses trabalhadores (MOURÃO, 2007).

Uma das consequências é revelada em um estudo realizado por Galvão e Sousa (2012) em Escolas Técnicas do SUS de distintas regiões do Brasil. Esses pesquisadores demonstraram que existe um escasso número de profissionais cedidos às escolas, assim como existe uma alta rotatividade entre os instrutores-supervisores que atuam nessas escolas. Em decorrência desses fatores há também dificuldades relacionadas a um maior aprofundamento do conceito de integração entre ensino-serviços por parte deles. As autoras ressaltam, no entanto, que apesar das lacunas encontradas foram percebidos o compromisso da equipe das instituições, o domínio que possuem os profissionais em sua área de atuação e o comprometimento do corpo docente, e de igual modo, a realização das capacitações pedagógicas que contribuem para o processo de descentralização dos serviços.

Já Vieira e Chinelli (2013), apontam os anos 2000, durante o mandato do Governo do Presidente Luís Inácio Lula da Silva, como um período de esperança, pela reorganização do Sistema. Todavia, as autoras ratificam que apesar de marcos importantes ${ }^{1}$, prevaleceu a inserção de políticas de forma fragmentada, com poucos avanços no reconhecimento dos profissionais de nível técnico, como por exemplo, alterações na carreira dos Agentes Comunitários de Saúde. Assim, a proposta de formação que foi pautada na história da formação técnica em saúde, abre espaço a um movimento presente na história da educação brasileira, que corresponde à formação do trabalhador aliada aos interesses do capital, mesmo nos serviços de saúde pública. Isso é um contrassenso posto que os serviços de saúde pública são garantidos constitucionalmente e não podem ser pensados ou guiados pelos direcionamentos do mercado capitalista.

Destaca-se que, no que diz respeito à formação dos trabalhadores da área da saúde, a Lei no 8.080, de 19 de setembro de 1990, que regulamenta os serviços de saúde em todo o território nacional, apresenta, em seu artigo 27 , inciso I que a formação dos trabalhadores deve se organizar no interior das instituições tendo em vista que: "um sistema de formação de recursos humanos em todos os níveis de ensino, inclusive de pós-graduação, além da elaboração de programas de permanente aperfeiçoamento de pessoal" (BRASIL, 1990).

\footnotetext{
1 Criação da Secretaria de Gestão do Trabalho e da Educação na Saúde vinculada ao Ministério da Saúde, em 2003. Esta secretaria tem como atribuição a formulação de políticas públicas no eixo da gestão, formação e, qualificação dos trabalhadores e da regulação profissional na área da saúde no Brasil.
} 
De acordo com informações disponibilizadas no site da Rede de Escolas Técnicas do Sistema Único de Saúde, existem 40 Escolas Técnicas do SUS espalhadas nas cinco regiões do país. Essas Escolas podem ofertar os seguintes cursos: Técnico de Nível Médio em Saúde Integrado ao Ensino Médio; Técnico em Agente Comunitário de Saúde; Técnico em Análises Clínicas; Técnico em Atendimento Pré-Hospitalar; Técnico em Citopatologia; Técnico em Enfermagem; Técnico em Farmácia; Técnico em Gerência em Saúde; Técnico em Hemoterapia; Técnico em Imobilização Ortopédica; Técnico em Nutrição e Dietética; Técnico em Órteses e Próteses; Técnico em Prótese Dentária; Técnico em Radiologia; Técnico em Reabilitação de Dependentes Químicos; Técnico em Registros e Informações em Saúde; Técnico em Saúde Bucal; Técnico em Segurança do Trabalho; Técnico em Vigilância em Saúde e Técnico Integrado ao Ensino Médio na Modalidade de Jovens e Adultos em Radiologia (ETSUS, 2016). Vê-se que há uma gama de cursos técnicos voltados para a qualificação dos trabalhadores em serviço e, sendo assim, estudos acerca dessa formação devem ser constantemente acompanhados com o intuito de compreender como ocorre a produção do conhecimento sobre a formação desses trabalhadores no país.

Dessa forma, buscando aprofundar a reflexão em torno da formação técnica em saúde verifica-se que à produção do conhecimento na área faz-se necessário resgatar princípios essenciais a essa formação. Isso porque

no processo de construção dessas capacidades e habilidades, é preciso propiciar uma formação que permita aos trabalhadores agir como cidadãos produtores de bens e de serviços e como atores na sociedade civil. Mais do que nunca, "a educação deve possibilitar aos trabalhadores [...] sua participação na sociedade científica e tecnológica não como objetos, mas como sujeitos, resgatando assim a dimensão política: a construção da identidade social e a integração plena na cidadania. (AMÂNCIO, 2004, p. 378 apud DELUIZ, 1997, p.16).

É preciso destacar também os desafios para a formação dos trabalhadores de nível técnico em saúde, considerando um modelo social excludente. Modelo que se propõe, muitas vezes, a reduzir a formação profissional dos trabalhadores de nível técnico a já existente e também excludente divisão técnica do trabalho em saúde, contribuindo para a manutenção da hegemonia do ideário cientificista e tecnicista na área (PEREIRA; LIMA, 2006). Para a Organização PanAmericana de Saúde,

o técnico em saúde é aquele profissional que realiza ações específicas e fundamentalmente sistematizadas no campo da saúde e toma decisões dentro da área de sua competência [...] executa suas funções sob supervisão direta ou indireta do pessoal de nível superior [...] a complexidade de suas ações é maior que a de profissionais auxiliares e menor que do pessoal de nível profissional ou equivalente (RETS, 2009, p.10).

Considera-se, nesse trabalho, a produção do conhecimento como um fator de interação e transformação das relações entre os indivíduos, pois a informação é transformada por meio de análises que refletem posicionamentos e escolhas dos pesquisadores. Assim, buscando refletir sobre a produção do conhecimento na área da formação técnica em saúde, foi utilizada como metodologia a revisão bibliográfica, dentro de uma abordagem qualitativa. Para tanto, foram selecionadas teses e dissertações presentes no repositório da Coordenação e Aperfeiçoamento de Pessoal de Nível Superior (CAPES): (www.periodicos.capes.gov.br). 
Optou-se por buscar os procedimentos metodológicos porque para Bergamo e Bernardes (2006) esses apresentam a existência de uma relação entre o pesquisador e o conteúdo de investigação, assim, o método científico envolve aspectos objetivos e subjetivos que serão materializados na produção científica em busca de tornar a exposição mais fidedigna. Para esses autores:

É possível perceber que tanto o ato de pesquisar quanto o objeto de pesquisa não são neutros, e que o ofício de pesquisa é um ato político, que só pode haver certos tipos de sujeito de conhecimento, certas ordens de verdade, certos domínios de saber a partir das condições que são o solo em que se formam o sujeito, os domínios de saber e as relações com a verdade. (BERGAMO E BERNARDES, 2006, p. 196).

Diante dessa perspectiva, buscar os procedimentos metodológicos adotados para as investigações acerca da formação técnica em saúde conduz à direção de compreender como a produção científica brasileira tem se utilizado desses procedimentos para analisar a educação profissional que vem sendo desenvolvida para o atendimento nos serviços em saúde.

\section{METODOLOGIA}

Para o desenvolvimento dessa pesquisa, foi feito um levantamento da produção do conhecimento sobre a formação técnica em saúde com o propósito de identificar os procedimentos metodológicos e os objetivos das dissertações e teses que foram produzidas no período de 2002 a 2016. Para tanto foi utilizado o Portal da CAPES, precisamente o Banco de Teses e Dissertações.

A pesquisa ocorreu no mês de agosto de 2016, especificamente no dia dezoito de agosto de 2016, cuja investigação identificou através dos parâmetros mencionados a seguir, as produções científicas que formam os resultados desse mapeamento e que serviram para a análise e discussão sobre a formação dos trabalhadores em saúde. Para o levantamento dos dados foi realizada uma busca com o descritor "Formação Técnica em Saúde" entre aspas duplas.

Os resultados da pesquisa foram organizados conforme a Figura 01 e a Figura 02. Em seguida foi feita a leitura dos trabalhos encontrados e extraída a metodologia e o objetivo de cada um desses a fim de compreender os critérios de pesquisa adotados e descritos em cada trabalho como procedimento metodológico.

A pesquisa identificou dezesseis trabalhos utilizando o termo Formação Técnica em Saúde, portanto, há no repositório consultado 16 produções de conhecimento acerca da temática. Deste total, seis, não estavam disponíveis no repositório de suas universidades, por essa razão, não constam na discussão dos resultados. Na Figura 1 identificam-se os trabalhos não encontrados. 
Figura 1: Quadro - Produções Acadêmicas identificadas e não encontradas sobre Formação Técnica em Saúde (2002-2016)

\begin{tabular}{|c|c|c|c|}
\hline № & Autor & Título do trabalho & Ano \\
\hline 01 & $\begin{array}{l}\text { CASTRO, } \\
\text { Gracinete } \\
\text { Rodrigues } \\
\text { de. }\end{array}$ & $\begin{array}{l}\text { Educação Profissional em Saúde: um estudo sobre a avaliação da } \\
\text { aprendizagem na Escola de Formação Técnica em Saúde Enfermeira } \\
\text { Izabel dos Santos (RJ). }\end{array}$ & 2016 \\
\hline 02 & $\begin{array}{l}\text { CARDOSO, } \\
\text { Marina } \\
\text { Garcia. }\end{array}$ & $\begin{array}{l}\text { O Agente Comunitário de Saúde na atenção à gestante e a puérpera: } \\
\text { repercussões de uma estratégia de educação permanente. }\end{array}$ & 2014 \\
\hline 03 & $\begin{array}{l}\text { AZEVEDO, } \\
\text { Anderson } \\
\text { Leonardo } \\
\text { De. }\end{array}$ & $\begin{array}{l}\text { Análise das condições de funcionamento das bibliotecas nas instituições } \\
\text { de Formação dos Trabalhadores Técnicos em Saúde nos Países Africanos } \\
\text { de Língua Oficial Portuguesa. }\end{array}$ & 2014 \\
\hline 04 & $\begin{array}{l}\text { Barbosa, } \\
\text { Marta de } \\
\text { Fátima Lima. }\end{array}$ & $\begin{array}{l}\text { Integralidade: Sentido, construção e aplicação para os técnicos de } \\
\text { enfermagem. }\end{array}$ & 2009 \\
\hline 05 & $\begin{array}{l}\text { RESENDE, } \\
\text { Patrícia } \\
\text { Policeno de. }\end{array}$ & $\begin{array}{l}\text { Formação Técnica em Saúde na ETSUS Tocantins: análise da relação de } \\
\text { integração do ensino com os serviços de saúde. }\end{array}$ & 2009 \\
\hline 06 & $\begin{array}{l}\text { BRASIL, } \\
\text { Isabel } \\
\text { Pereira. }\end{array}$ & $\begin{array}{l}\text { A Formação Profissional em serviço no cenário do Sistema Único de } \\
\text { Saúde. }\end{array}$ & 2002 \\
\hline
\end{tabular}

\section{RESULTA DOS E DISCUSSÕES}

Dos 16 trabalhos identificados no Portal da Capes, apenas 10 foram encontrados na íntegra e selecionados para discussão. Esses apontam para uma variedade de produções em diversos Programas de Pós-Graduação no Brasil. Destacando-se dentre os achados seis produções, que foram oriundas de Mestrados Profissionais. Esses resultantes da ampliação do acesso aos mestrados que auxiliam na articulação entre a universidade e a realidade social (NEGRET, 2008). As produções das dissertações que serão discutidas foram realizadas: na Região Nordeste (4 produções), Sudeste (5 produções) e Sul (1 produção). No que diz respeito às teses com o parâmetro de busca já apresentado, foi encontrada apenas uma produção na Região Sul do país, conforme apresentadas na Figura 2. 
FIGURA 2: QUADRO - PRODUÇÕES ACADÊMICAS SOBRE FORMAÇÃO TÉCNICA EM SAÚDE (2007-2014).

No REFERÊNCIAS

01 AQUINO, Marina Garcia Cardoso de. 0 Agente Comunitário de Saúde na atenção à gestante e à puérpera: repercussões e uma estratégia de Educação Permanente / 2014. 67 p.

Dissertação (mestrado profissional) Instituto de Saúde

Coletiva. Universidade Federal da Bahia.

\section{PROCEDIMENTOS METODOLÓGICOS}

O estudo é de avaliação dos efeitos do Curso de Atualização para Agentes Comunitários de Saúde na atenção à saúde da mulher no ciclo gravídicopuerperal. Para coleta de dados foram utilizadas múltiplas técnicas, tais como: Análise documental, Entrevistas semiestruturadas com os Agentes Comunitários de Saúde, gestantes, puérperas, e enfermeiro afim de caracterizar como se dá o acompanhamento das usuárias no ciclo gravídico puerperal.

Trata-se de uma pesquisa qualitativa desenvolvida em um estudo de caso exploratório e descritivo. Os dados foram colhidos por meio de pesquisa documental e de entrevistas com informantes-chave. O lócus do estudo foi a Escola Técnica em Saúde Prof. Jorge Novís. A sistematização dos dados foi em matrizes de análise, onde foram confrontados os resultados das entrevistas e dos documentos com os objetivos e as diretrizes do Programa de Formação de Profissionais de Nível Médio para a Saúde.

03 MACHADO, Maria Élida.

Tensões entre as concepções e as políticas públicas de Educação Profissional em Saúde de nível médio no Estado do Rio Grande do Sul /2013. 142 f.Tese (Doutorado) Universidade do Estado do Rio de Janeiro. Faculdade de Educação.
O estudo busca apreender as tensões de natureza conceptual, política e legal, historicamente construídas, na relação entre as necessidades de formação técnica em saúde e políticas educacionais, identificando implicações sobre a regulação da Educação Profissional em Saúde no Estado do Rio Grande do Sul é o objetivo do trabalho. Foi desenvolvido por meio da análise de documentos legais e escuta dos agentes formuladores das políticas de Educação Profissional em Saúde, membros dos Conselhos de Saúde e de Educação do Estado do Rio Grande do Sul.

A pesquisa é um estudo de avaliabilidade do Curso de Habilitação Profissional de Técnico em Saúde Bucal, desenvolvido pela Escola de Formação Técnica em Saúde Prof. Jorge Novis, unidade da Superintendência 
Dissertação (Mestrado Profissional em Gestão de Sistemas de Saúde) 0 Universidade Federal da Bahia - Instituto de Saúde Coletiva. de Recursos Humanos da Secretaria da Saúde do Estado da Bahia. Foram realizadas doze entrevistas e a observação in loco, nos momentos de dispersão do curso com os cirurgiões dentistas, sendo um coordenador, instrutores e com os beneficiários do curso, os futuros Técnicos de Saúde Bucal.

05 MODESTO, Maria do Socorro Andrade. Formação técnica versus prática: percepção dos agentes comunitários de saúde do Tocantins. Dissertação (Mestrado em Saúde e Gestão do Trabalho) - Programa de Mestrado Profissional em Saúde e Gestão do Trabalho, Universidade do Vale do Itajaí, Itajaí, 2010.

06 RIBEIRO, Nélia Beatriz Caiafa.

Dimensões do cuidado: um estudo sobre a formação de técnicos em higiene dental.145 f. Dissertação (Mestrado Profissional em Saúde). Rio de Janeiro, 2010.

07 CAMARÃO, Maria José Côrtes. Currículos e competências: experiência docente nos cursos técnicos em saúde. 2010. 76 f. Dissertação (Mestrado profissionalizante) - Instituto de Saúde Coletiva. Universidade Federal da Bahia.

08 GOLDSCHMIDT, Irene Leonore. Arte e saúde: o teatro na educação em saúde. 134 f. Dissertação (Mestrado Profissional em Educação Profissional em Saúde) Escola Politécnica de Saúde Joaquim Venâncio - Fundação Oswaldo Cruz, 2010.
O estudo se deu sob uma abordagem qualitativa, tomando como sujeitos da pesquisa egressos do curso para formação de Técnico em Higiene Dental realizado em Nova Iguaçu, no período de agosto de 2006 a junho de 2007. Como técnicas de pesquisa foram adotadas a realização de grupo focal, entrevistas semiestruturadas com egressos do curso e entrevistas com cirurgiões-dentistas, além da revisão bibliográfica de concepções de educação/educação profissional e cuidado em saúde para construir o referencial teórico. $O$ tratamento do material obtido no trabalho de campo foi orientado pela Análise de Conteúdo, sob a modalidade Análise Temática. mudanças desencadeadas, nas práticas docentes, pelo processo de construção dos currículos nos cursos técnicos desenvolvidos pela EFTS-Bahia. Esse trabalho utilizou fontes primárias, entrevista semiestruturada com informantes-chaves e fontes secundárias através da pesquisa documental. Participaram como sujeitos dessa pesquisa, os formadores dos cursos técnicos de enfermagem da Escola de Formação Técnica em Saúde do SUS Bahia.

O estudo desenvolve revisão bibliográfica referenciada no materialismo histórico e dialético, discutindo o processo de conhecimento e suas relações com a racionalidade científica, ressaltando a importância da percepção e da sensibilidade. Analisa o desenvolvimento dos Estudos Culturais na Inglaterra, que apontam para o conceito de cultura como produção social de uma coletividade. Pesquisa em Adorno e Benjamim o papel da arte nas sociedades capitalistas, o desenvolvimento da
Trata-se de um estudo de caso que analisa as 
indústria cultural e a alienação do artista. Estabelece relações entre estética e poder no capitalismo. A autora objetivava levantar possibilidades e implicações da utilização do teatro para a educação dos trabalhadores em saúde.

09 BITTAR, Sandra Ferreira Gesto. A formação profissional do auxiliar de enfermagem: a experiência do Projeto Larga Escala no município de Natividade/RJ. $81 \mathrm{f}$. Dissertação (Mestrado apresentado Escola Nacional de Saúde Pública Sérgio Arouca), Rio de Janeiro, 2009
A pesquisa foi realizada em documentos na ETISSESDEC/RJ e aplicado um questionário com perguntas abertas e fechadas às egressas do curso. Os dados foram trabalhados dentro de uma abordagem quantiqualitativa. Este trabalho teve como objeto de estudo - Curso de Formação Profissional de Auxiliar de Enfermagem realizado no município de Natividade, estado do Rio de Janeiro nos anos de 1995 a 1997 e teve como objetivo estudar os efeitos ocorridos na prática profissional das egressas da rede de saúde.

10 MARQUES, Cláudia Maria da Silva. As competências crítico-emancipatórias e a formação dos trabalhadores de nível médio em enfermagem: focalizando as políticas ministeriais. São Paulo, 2007. 91 p. Dissertação (Mestrado) - Escola de Enfermagem da Universidade de São Paulo.

Pesquisa descritiva e exploratória, na perspectiva
qualitativa. O suporte teórico foi a Teoria de
Intervenção Práxica da Enfermagem em Saúde
Coletiva; o referencial de análise foi a hermenêutica-
crítica. A empiria foi constituída por documentos
reguladores da educação profissional técnica
oriundos dos Ministérios citados, entre os anos 1996
e 2006 . Além disso, foram levantados os discursos de
seus representantes, através de entrevista
semiestruturada.

Pesquisa descritiva e exploratória, na perspectiva qualitativa. O suporte teórico foi a Teoria de Intervenção Práxica da Enfermagem em Saúde Coletiva; o referencial de análise foi a hermenêuticacrítica. A empiria foi constituída por documentos reguladores da educação profissional técnica oriundos dos Ministérios citados, entre os anos 1996 e 2006. Além disso, foram levantados os discursos de semiestruturada.

Considerando o objetivo específico deste trabalho, que é verificar a metodologia utilizada pelos autores das produções referenciadas, para o alcance do objetivo da pesquisa, na Figura 2 constata-se como principais procedimentos metodológicos de pesquisa: a revisão bibliográfica, a análise de fontes primárias - documentos institucionais, tendo como principal formato o estudo de caso. A seguir, serão apresentados comentários acerca das produções encontradas e suas metodologias.

Aquino (2014) buscando analisar os efeitos do curso de atualização das práticas dos Agentes Comunitários de Saúde; um curso de atualização na atenção à saúde da mulher, desenvolvido pela Escola de Formação Técnica em Saúde do Estado da Bahia, utilizou análise documental e entrevistas semiestruturadas com agentes comunitários de saúde, enfermeiros e usuárias gestantes ou puérperas. Esse teve

como informantes chave da pesquisa foram identificados oito agentes comunitários de saúde da unidade selecionada que haviam feito o curso de atualização, uma enfermeira da unidade docente do curso e quatro mulheres no ciclo gravídico puerperal da área adscrita contactadas na residência ou no momento da consulta (2014, p.22).

A pesquisa de Costa (2014) realiza uma pesquisa qualitativa, do tipo estudo de caso, exploratório, descritivo. Para produção dos dados foi realizada análise documental e entrevistas com informantes-chave. Tal pesquisa buscou investigar a implementação do Programa de Formação 
de Profissionais de Nível Médio para a Saúde na Bahia de 2010 a 2013, identificando facilitadores e obstrutores desse processo. Como justificativa do caminho escolhido a autora aborda que

o modelo busca favorecer a visualização das ações a serem priorizadas, as estratégias para implementação, o objetivo do programa, assim como as instituições envolvidas na criação e implementação da política. (2014, p. 28).

A tese construída por Machado (2012) buscou apreender as tensões de natureza conceptual, política e legal, historicamente construídas, na relação entre as necessidades de formação técnica em saúde e políticas educacionais, identificando implicações sobre a regulação da Educação Profissional em Saúde no Estado do Rio Grande do Sul, por meio da análise de documentos legais e escuta dos agentes formuladores das políticas de Educação profissional em Saúde, membros dos Conselhos de Saúde e de Educação do Estado do Rio Grande do Sul. A autora buscou "a análise das políticas de saúde e de educação procurou apreender as tensões, construídas historicamente, entre essas políticas" $(2012$, p.23).

Mazzafera (2010) buscou realizar uma pesquisa de avaliabilidade do Curso de Habilitação Profissional de Técnico em Saúde Bucal, desenvolvido pela Escola de Formação Técnica em Saúde Prof. Jorge Novis da Superintendência de Recursos Humanos da Secretaria da Saúde do Estado da Bahia, tendo como objetivo identificar pontos para sua melhoria e focos para avaliação futura. Como estratégia de investigação a autora desenvolveu

[...] um estudo de avaliação dos efeitos do Curso de Atualização para Agentes Comunitários de Saúde (ACS) na atenção à saúde da mulher no ciclo gravídico-puerperal, em uma Unidade de Saúde da Família (USF) localizada no Distrito Sanitário de Cajazeiras, no município de Salvador no ano de 2014 (2014, p.21).

Modesto (2010) assenta-se em uma pesquisa quantitativa, do tipo exploratória e de campo por meio da utilização de um questionário adaptado e modificado por Luiz (1999), em um estudo realizado pela Universidade Federal de São Carlos - SP, que tinha como objetivo apreender a percepção dos egressos técnicos de Ensino Médio. Modesto (2010) teve como objetivo verificar a percepção de uma amostra de Agentes Comunitários de Saúde do município de Palmas.

O procedimento metodológico que se adotou para coleta de dados desta investigação foi por meio do preenchimento de um questionário adaptado e modificado por Luiz (1999), o qual foi respondido por uma amostra de egressos da Escola Técnica de Saúde do Tocantins - ETSUS/TO. (2010, p.48).

Ribeiro (2010) em busca de atingir o objetivo do seu estudo, se propõe revisar o referencial teórico que embasa a proposta pedagógica da Escola Técnica Isabel dos Santos, fundamentada numa concepção de educação que implica ação e reflexão dos homens sobre o mundo para transformá-lo (Ribeiro, p.59 2010) utilizou-se como método: grupo focal e entrevistas semiestruturadas. Para o tratamento do material obtido por intermédio das entrevistas usa a Análise de Conteúdo, sob a modalidade Análise Temática (2010, p. 19). Os sujeitos da pesquisa são egressos do curso de Formação de Técnico em Higiene Dental e seus supervisores. O curso foi realizado em Nova Iguaçu, no período entre agosto de 2006 a junho de 2007. 
A pesquisa de Camarão (2010) utiliza como método o estudo de caso. Como fontes primárias, utiliza a entrevista semiestruturada com informantes chaves e fontes secundárias através da pesquisa documental para analisar as mudanças desencadeadas, nas práticas docentes, pelo processo de construção dos currículos nos cursos técnicos desenvolvidos pela Escola de Formação Técnica do SUS -Bahia. Como complemento à sua pesquisa, a autora utilizou a técnica "da observação participante [...] esta experiência pode contribuir na acessibilidade das informações, na pesquisa documental das propostas implementadas no passado e atualmente" (2010, p.19).

O estudo realizado por Goldschmidt (2010) aponta para uma revisão bibliográfica referenciada no materialismo histórico e dialético, buscando o conceito de cultura como produção social de uma coletividade. Essa autora tinha como objetivo levantar possibilidades e implicações da utilização do teatro para a educação dos trabalhadores. Essa utiliza também a entrevista como técnica, para captar, a partir da experiência de sujeitos diversos escolhidos: "[...] a relação entre teatro, educação e em alguns casos, saúde, que questões consideram relevantes na utilização do teatro como prática pedagógica" (2000, p.14).

Bittar (2009), por sua vez utilizou-se da pesquisa documental na Escola de Formação Técnica em Saúde Enfermeira Izabel dos Santos da Secretaria do Estado de Saúde e Defesa Civil/ RJ ETISSESDEC/RJ, bem como foi utilizado questionário com perguntas abertas e fechadas às egressas do curso de Técnico de Enfermagem formadas nos anos de 1995 a 1997, pela Escola de Formação Técnica em Saúde Enf.a Izabel dos Santos. Os dados foram trabalhados dentro de uma abordagem quanti-qualitativa. Tal pesquisa foi realizada no município de Natividade, estado do Rio de Janeiro. Nesse município foi implantado e desenvolvido o Projeto Larga Escala. A autora aborda que um dos facilitadores da sua pesquisa ocorreu pelo fato de "na aplicação dos questionários a maioria das respondentes permanece morando e trabalhando em Natividade ou em municípios limítrofes" (2009, p.50).

Marques (2007) apresenta um estudo de caráter descritivo e exploratório, na perspectiva qualitativa, que busca identificar a noção de competência assumida pelos Ministérios da Educação e da Saúde. Para justificar a escolha metodológica do seu trabalho, a autora apresenta em sua discussão que o trabalho foi

apoiado no estudo de Queiroz, Egry (1988), que propõem as seguintes fases: captação da realidade objetiva; interpretação da realidade objetiva; construção do projeto de intervenção na realidade objetiva; intervenção na realidade objetiva; reinterpretação da realidade objetiva. (2007, p.20).

Frente ao exposto, observa-se que os estudos apresentados se dedicam a análise e avaliação da formação dos trabalhadores de nível técnico oriundos de instituições vinculados às escolas que integram a Rede de Escolas Técnicas do Sistema Único de Saúde. Isso contempla o objetivo específico que visa além de caracterizar os procedimentos metodológicos adotados nessas produções para compreender a temática construída em torno dessa formação, visualizar aqueles estudos que têm como campo empírico as Escolas Técnicas de Saúde. Destaca-se que dos estudos apresentados somente Marques (2007) e Machado (2013), portanto, dois trabalhos, não apresentam essas escolas como campo de estudo.

Ao tratar de estudos que se utilizam de análise qualitativa, Minayo parte da seguinte reflexão: "o verbo principal da análise qualitativa é compreender" (2011, p.623). Assim, ratificando estas palavras, entende-se a prevalência do uso de métodos qualitativos a partir da pretensão demonstrada nos objetivos dos estudos discutidos nesse trabalho, nos conduzindo a compreender 
que os procedimentos metodológicos adotados na produção encontrada foram suficientes à compreensão do que estava em investigação no espaço de formação dos profissionais de nível técnico que atuam na saúde.

\section{CONCLUSÃO}

A pesquisa apresentou a produção do conhecimento acerca da formação dos trabalhadores de nível técnico que vem acontecendo de maneira considerável nos últimos anos, tendo em vista que foram produzidos dezesseis trabalhos no período elencado (2002 a 2016). Destaca-se a grande produção desta temática vinda dos mestrados profissionais, que refletem a real necessidade de uma maior atenção aos trabalhadores de nível técnico, considerando que estes estão diretamente ligados ao cotidiano dos serviços de saúde.

Os achados dessa pesquisa apresentam o interesse dos pesquisadores pela formação dos profissionais de nível técnico, bem como a avaliação e reconhecimento do seu trabalho pelos demais profissionais do serviço, necessitando, portanto, ampliar as discussões que contribuam para seu reconhecimento. Com a discussão da ampliação da produção do conhecimento sobre a temática, vale destacar as dificuldades encontradas para o acesso das produções que foram desenvolvidas em instituições privadas. Tais instituições não possuem repositórios de teses e dissertações abertos a membros externos, dificultando a disseminação do conhecimento.

Os procedimentos metodológicos encontrados nos trabalhos apresentam em sua maioria, teor qualitativo, constando: três estudos de caso, três pesquisas bibliográficas e quatro pesquisas envolvendo abordagens metodológicas avaliativas.

Chama-se a atenção à organização das produções acadêmicas sobre a temática em estudo no país, tendo a região Sudeste com maior quantidade de trabalhos publicados, seguida da região Nordeste com quatro publicações e Sul com uma publicação. Destoa a ausência de produção nas demais regiões do país (Norte e Centro-Oeste), tendo em vista o grande quantitativo de Escolas Técnicas do Sistema Único de Saúde no Brasil e nessas regiões, podendo ser tomado como um indicador de uma maior precarização do trabalho destes profissionais nestas regiões ou como desinteresse dos pesquisadores em investigar esses espaços. Fica, então, o registro da necessidade de pesquisas acerca da temática nessas regiões.

\section{REFERÊNCIAS}

1. AMANCIO FILHO, Antenor. Dilemas e desafios da formação profissional em saúde. Interface (Botucatu), Botucatu, v. 8, n. 15, p. 375-380, agosto. 2004.

2. AQUINO, Marina Garcia Cardoso de. O Agente Comunitário de Saúde na atenção à gestante e à puérpera: repercussões e uma estratégia de Educação Permanente / 2014. 67 p.Dissertação (mestrado profissional) - Instituto de Saúde Coletiva. Universidade Federal da Bahia. 
3. AZEVEDO, ANDERSON LEONARDO DE. Análise das condições de funcionamento das bibliotecas nas instituições de Formação dos Trabalhadores Técnicos em Saúde nos Países Africanos de Língua Oficial Portuguesa' 29/02/2016 124 f. Mestrado Profissional em EDUCAÇÃO PROFISSIONAL EM SAÚDE Instituição de Ensino: FUNDACAO OSWALDO CRUZ, Rio de Janeiro Biblioteca Depositária: Emília Bustamante

4. BARBOSA, Marta de Fátima Lima. INTEGRALIDADE: Sentido, construção e aplicação para os técnicos de enfermagem.' 01/11/2009 75 f. Profissionalizante em SAÚDE PÚBLICA Instituição de Ensino: FUNDACAO OSWALDO CRUZ, RIO DE JANEIRO Biblioteca Depositária: Lincoln de Freitas Filho.

5. BERGAMO, G. A.; BERNARDES, Marisa Rezende. Produção de conhecimento. Educ. Soc., Campinas, v. 27, n. 94, p. 179-198, abril. 2006.

6. BITTAR, Sandra Ferreira Gesto. A formação profissional do auxiliar de enfermagem: a experiência do Projeto Larga Escala no município de Natividade/RJ. 81 f. Dissertação (Mestrado apresentado Escola Nacional de Saúde Pública Sérgio Arouca), Rio de Janeiro, 2009

7. BRASIL, Isabel Pereira. A Formação Profissional em Serviço no Cenário do Sistema Único de Saúde' 01/07/2002 254 f. Doutorado em EDUCAÇÃO: HISTÓRIA, POLÍTICA, SOCIEDADE Instituição de Ensino: PONTIFÍCIA UNIVERSIDADE CATÓLICA DE SÃO PAULO, SÃO PAULO Biblioteca Depositária: PUC-SP.

8. BRASIL, LEI № 8.080, DE 19 DE SETEMBRO DE 1990. Dispõe sobre as condições para a promoção, proteção e recuperação da saúde, a organização e o funcionamento dos serviços correspondentes e dá outras providências. Diário Oficial, Brasília, 19.set. 1990a.

9. CAMARÃO, Maria José Côrtes. Currículos e competências: experiência docente nos cursos técnicos em saúde. 2010. 76 f. Dissertação (Mestrado profissionalizante) - Instituto de Saúde Coletiva. Universidade Federal da Bahia.

10. CARDOSO, MARINA GARCIA. O AGENTE COMUNITÁRIO DE SAÚDE NA ATENÇÃO À GESTANTE E A PUÉRPERA: REPERCUSSÕES DE UMA ESTRATÉGIA DE EDUCAÇÃO PERMANENTE.' 30/09/2014 67 f. Mestrado Profissional em SAÚDE COLETIVA Instituição de Ensino: UNIVERSIDADE FEDERAL DA BAHIA, Salvador Biblioteca Depositária: Biblioteca Universitária de Saúde

11. CASTRO, GRACINETE RODRIGUES DE. EDUCAÇÃO PROFISSIONAL EM SAÚDE: UM ESTUDO SOBRE A AVALIAÇÃO DA APRENDIZAGEM NA ESCOLA DE FORMAÇÃO TÉCNICA EM SAÚDE ENFERMEIRA IZABEL DOS SANTOS (RJ)' 29/03/2016 undefined f. Mestrado Profissional em EDUCAÇÃO PROFISSIONAL EM SAÚDE Instituição de Ensino: FUNDACAO OSWALDO CRUZ, Rio de Janeiro Biblioteca Depositária: undefined

12. CASTRO, J. L. de. Protagonismo silencioso: a presença da OPAS na formação de recursos humanos em saúde no Brasil. Natal - RN: Observatório RH - NESC UFRN; Ministério da Saúde; OPAS/OMS, 2008. 267p.

13. COSTA, Claudia Wanderley Cruz e. Implementação de políticas nacionais para formação técnica em saúde no Estado da Bahia: o caso do PROFAPS / 2014. 75f. Dissertação (mestrado profissional) - Instituto de Saúde Coletiva. Universidade Federal da Bahia. 
14. ESCOLA Politécnica de Saúde Joaquim Venâncio. Caminhos da politécnica: 30 anos da Escola Politécnica de Saúde Joaquim Venâncio / Organização Escola Politécnica de Saúde Joaquim Venâncio. - Rio de Janeiro: EPSJV, 2016.

15. GALVAO, E. de A.; SOUSA, M. F. de. As escolas técnicas do SUS: que projetos políticopedagógicos as sustentam? Physis, Rio de Janeiro, v. 22, n. 3, p. 1159-1189, 2012

16. GOLDSCHMIDT, Irene Leonore. ARTE E SAÚDE: 0 teatro na educação em saúde. 2010 134 f. Dissertação (Mestrado Profissional em Educação Profissional em Saúde) - Escola Politécnica de Saúde Joaquim Venâncio - Fundação Oswaldo Cruz.

17. MACHADO, Maria Élida. Tensões entre as concepções e as políticas públicas de Educação Profissional em Saúde de nível médio no Estado do Rio Grande do Sul /2013. 142 f.Tese (Doutorado) Universidade do Estado do Rio de Janeiro. Faculdade de Educação.

18. MARQUES, Cláudia Maria da Silva. As competências crítico-emancipatórias e a formação dos trabalhadores de nível médio em enfermagem: focalizando as políticas ministeriais. São Paulo, 2007. 91 p. Dissertação (Mestrado) - Escola de Enfermagem da Universidade de São Paulo.

19. MAZZAFERA, Leila Maria Coutinho. 0 curso de habilitação profissional do técnico em saúde bucal no âmbito do SUS/BA: um estudo sobre avaliabilidade. 75 f. 2010. Dissertação (Mestrado Profissional em Gestão de Sistemas de Saúde) 0 Universidade Federal da Bahia - Instituto de Saúde Coletiva.

20. MINAYO, Maria Cecília de Souza. Análise qualitativa: teoria, passos e fidedignidade. Ciênc. saúde coletiva, Rio de Janeiro, v. 17, n. 3, p. 621-626, Mar. 2012.

21. MODESTO, Maria do Socorro Andrade. Formação técnica versus prática: percepção dos agentes comunitários de saúde do Tocantins. Dissertação (Mestrado em Saúde e Gestão do Trabalho) - Programa de Mestrado Profissional em Saúde e Gestão do Trabalho, Universidade do Vale do Itajaí, Itajaí, 2010.

22. MOURÃO, A. M. A. et al. A formação dos trabalhadores sociais no contexto neoliberal. $O$ projeto das residências em saúde da Faculdade de Serviço Social da Universidade Federal de Juiz de Fora. In: MOTA, A. E. et al. (Org.). Serviço social e saúde: formação e trabalho profissional. 2. ed. São Paulo: Cortez, 2007. p. 352-380

23. NEGRET, F. A identidade e a importância dos mestrados profissionais no Brasil e algumas considerações para a sua avaliação. Revista Brasileira Pós-Graduação, Brasília, v. 5, n. 10, p. 217-225, dezembro de 2008.

24. PEREIRA, I. B; LIMA, J. C. F. Educação profissional em saúde. In: BRASIL, I.; LIMA, J. C. F. (Orgs). Dicionário da educação profissional em saúde. Rio de Janeiro: Fiocruz, 2006

25. PRONKO, M. A formação de trabalhadores técnicos em saúde no Brasil e no Mercosul- Rio de Janeiro: EPSJV, 2011. 302 p.

26. RESENDE, Patrícia Policeno de. FORMAÇÃO TÉCNICA EM SAÚDE NA ETSUS TOCANTINS: análise da relação de integração do ensino com os serviços de saúde.' 01/11/2009 $150 \mathrm{f}$. Profissionalizante em SAÚDE Instituição de Ensino: UNIVERSIDADE DO VALE DO ITAJAÍ, Itajaí Biblioteca Depositária: UNIVALI 
27. REVISTA RETS. Secretaria Executiva da RETS, Rio de Janeiro: v. 1, n. 2, fev./abr. 2009.

28. RIBEIRO, Nélia Beatriz Caiafa. Dimensões do cuidado: um estudo sobre a formação de técnicos em higiene dental.145 f. Dissertação (Mestrado Profissional em Saúde). Rio de Janeiro, 2010.

29. VIEIRA, M.; CHINELLI, F. Relação contemporânea entre trabalho, qualificação e reconhecimento: repercussões sobre os trabalhadores técnicos do SUS. Ciênc. Saúde coletiva, Rio de Janeiro, v. 18, n. 6, p. 1591-1600, Junho, 2013. 